\title{
Cycles in folded hypercubes
}

\author{
Jun-Ming $\mathrm{Xu}^{*}$, Meijie Ma \\ Department of Mathematics, University of Science and Technology of China, Hefei, Anhui, 230026, China
}

Received 6 February 2004; received in revised form 4 February 2005; accepted 17 April 2005

\begin{abstract}
This work investigates important properties related to cycles of embedding into the folded hypercube $F Q_{n}$ for $n \geq 2$. The authors observe that $F Q_{n}$ is bipartite if and only if $n$ is odd, and show that the minimum length of odd cycles is $n+1$ if $n$ is even. The authors further show that every edge of $F Q_{n}$ lies on a cycle of every even length from 4 to $2^{n}$; if $n$ is even, every edge of $F Q_{n}$ also lies on a cycle of every odd length from $n+1$ to $2^{n}-1$.

(C) 2005 Elsevier Ltd. All rights reserved.

MSC: $05 \mathrm{C} 40$

Keywords: Folded hypercube; Pancyclic; Edge-pancyclic; Interconnection networks

\section{Introduction}

As a topology for an interconnection network of a multiprocessor system, the hypercube structure is a widely used and well-known interconnection model since it possesses many attractive properties $[1,2]$. The $n$-dimensional hypercube $Q_{n}$ is a graph with $2^{n}$ vertices, each vertex with a distinct binary string $x_{1} x_{2} \cdots x_{n}$ on the set $\{0,1\}$. Two vertices are linked by an edge if and only if their strings differ in exactly one bit. We use the symbol $d_{H}(x, y)$ to denote the Hamming distance between two vertices $u$ and $v$ in $Q_{n}$, that is, the number of different bits in the corresponding strings of both vertices. Clearly, $d_{H}(x, y)=d_{Q_{n}}(x, y)$, where $d_{Q_{n}}(x, y)$ denotes the distance between two vertices $x$ and $y$ in $Q_{n}$, i.e., the length of the shortest $x y$-path in $Q_{n}$.
\end{abstract}

\footnotetext{
This work was supported partially by NNSF of China (No. 10271114)

* Corresponding author.

E-mail address: xujm@ustc.edu.cn (J.-M. Xu).
} 


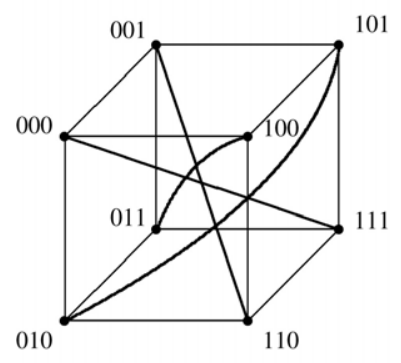

$\mathrm{FQ}_{3}$

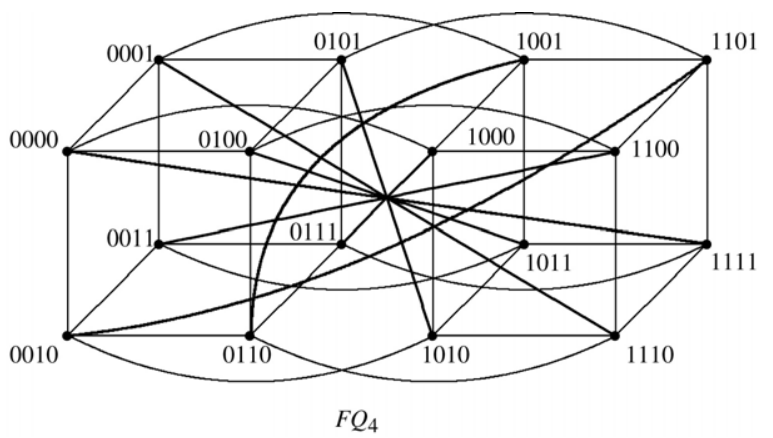

Fig. 1. $F Q_{3}$ and $F Q_{4}$. (Thick lines represent the complementary edges.)

As a variant of the hypercube, the $n$-dimensional folded hypercube $F Q_{n}$, proposed first by El-Amawy and Latifi [3], is a graph obtained from the hypercube $Q_{n}$ by adding an edge, called a complementary edge, between any two vertices $x=\left(x_{1} x_{2} \cdots x_{n}\right)$ and $\bar{x}=\left(\bar{x}_{1} \bar{x}_{2} \ldots \bar{x}_{n}\right)$, where $\bar{x}_{i}=1-x_{i}$. The graphs shown in Fig. 1 are $F Q_{3}$ and $F Q_{4}$, respectively.

It has been shown that $F Q_{n}$ is $(n+1)$-regular $(n+1)$-connected. $F Q_{n}$ is also superior to $Q_{n}$ in some properties. For example, it has diameter $\left\lceil\frac{n}{2}\right\rceil$, about half the diameter of $Q_{n}$ [3]. Thus, the folded hypercube $F Q_{n}$ is an enhancement on the hypercube $Q_{n}$. In particular, there are $n+1$ internally disjoint paths of length at most $\left\lceil\frac{n}{2}\right\rceil+1$ between any pair of vertices in $F Q_{n}$, the deletion of less than $\left\lceil\frac{n}{2}\right\rceil-2$ vertices or edges does not increase the diameter of $F Q_{n}$, and the deletion of up to $n$ vertices or edges increases it by at most one [4,5]. These properties mean that interconnection networks modelled by $F Q_{n}$ are extremely robust. As a result, the study of the folded hypercube has recently attracted the attention of many researchers [6-8].

In [9], Li et al. proved that every edge of $Q_{n}(n \geq 2)$ lies on a cycle of every even length from 4 to $2^{n}$. In this work, we first observe that $F Q_{n}$ is bipartite if and only if $n$ is odd, and show that the minimum length of even cycles in $F Q_{n}$ is 4 and that the minimum length of odd cycles is $n+1$ if $n$ is even. Then, using the $\mathrm{Li}$ et al. result, we further show that every edge of $F Q_{n}$ lies on a cycle of every even length from 4 to $2^{n}$; moreover, every edge of $F Q_{n}$ also lies on a cycle of odd length from $n+1$ to $2^{n}-1$ if $n$ is even.

The proofs of our main results are in Section 3. In the next section, we explore some new topological properties of $F Q_{n}$.

\section{New properties of $F Q_{n}$}

A vertex $u$ in $Q_{n}$ is said to be odd or even if the sum of its bits is odd or even. Let $X=\{u: u$ is odd $\}$ and $Y=\{u: u$ is even $\}$. Then $\{X, Y\}$ is a bipartition of $Q_{n}$, clearly. Conversely, if two vertices in $Q_{n}$ are in the same part of a bipartition of $Q_{n}$, then they have the same parity.

Theorem 2.1. $F Q_{n}$ is a bipartite graph if and only if $n$ is odd.

Proof. Since $F Q_{n}$ is obtained from $Q_{n}$ by adding $2^{n-1}$ complementary edges, to prove the theorem, it is sufficient to consider complementary edges. Let $\{X, Y\}$ be a bipartition of $Q_{n}$. Since any vertex $u$ and its complement $\bar{u}$ in $Q_{n}$ have different parity if and only if $n$ is odd and, hence, any complementary edge 
in $F Q_{n}$ joins two vertices in different parts of $\{X, Y\}$, it follows that $F Q_{n}$ is a bipartite graph if and only if $n$ is odd. The theorem follows.

Theorem 2.2. The length of any cycle in $F Q_{n}$ that contains exactly two complementary edges is even.

Proof. Let $C=\left(v, \bar{v}, v_{1}, \ldots, v_{m}, \bar{u}, u, u_{1}, \ldots, u_{n}, v\right)$ be a cycle in $F Q_{n}$ that contains exactly two complementary edges $e_{1}=(u, \bar{u})$ and $e_{2}=(v, \bar{v})$. Clearly, $v \neq u$ and, hence, $\bar{v} \neq \bar{u}$ since $e_{1} \neq e_{2}$.

If $F Q_{n}$ is bipartite, then we are done. We now assume $F Q_{n}$ is not bipartite. By Theorem $2.1, n$ is even and, hence, $u$ and $\bar{u}$ have the same parity. Let $\{X, Y\}$ be a bipartition of $Q_{n}$. Then, $u$ and $\bar{u}$ are in the same part of $\{X, Y\}$. Similarly, $v$ and $\bar{v}$ are also in the same part of $\{X, Y\}$. Since $C$ contains exactly two complementary edges, all of the other edges in $C$ are in $Q_{n}$. If $u, \bar{u}, v, \bar{v}$ are in the same part, then the lengths of the sections $\left(\bar{v}, v_{1}, \ldots, v_{m}, \bar{u}\right)$ and $\left(u, u_{1}, \ldots, u_{n}, v\right)$ are even. If $u, \bar{u}, v, \bar{v}$ are not in the same part, then the lengths of the two sections are odd. It follows that the length of $C$ is even in both cases.

Theorem 2.3. The length of any cycle in $F Q_{n}$ that contains exactly one complementary edge is at least $n+1$. Moreover, any complementary edge and any vertex lie on a common cycle of length $n+1$ containing the unique complementary edge in $F Q_{n}$.

Proof. Assume that $e=(u, \bar{u})$ is a complementary edge in $F Q_{n}$ and that $C$ is a cycle in $F Q_{n}$ containing exactly one complementary edge $e$. Then $P=C-e$ is a $u \bar{u}$-path in $Q_{n}$. Clearly, $d_{Q_{n}}(u, \bar{u})=n$ and, hence, the length of any $u \bar{u}$-path in $Q_{n}$ is at least $n$. Thus, the length of $P$ is at least $n$ and so the length of the cycle $C$ is at least $n+1$.

Let $P^{\prime}$ be a shortest $u \bar{u}$-path in $Q_{n}$ and let $v$ be any vertex in $F Q_{n}$. If $v \in\{u, \bar{u}\}$, then $P^{\prime}+e$ is a cycle of length exactly $n+1$ and contains the unique complementary edge $e$ in $F Q_{n}$. We now assume $v \notin\{u, \bar{u}\}$. Since $d_{H}(u, v)+d_{H}(\bar{u}, v)=n$, there are a shortest $v u$-path $R$ and a shortest $v \bar{u}$-path $R^{\prime}$ in $Q_{n}$. Then the sum of their lengths is equal to $n$. Clearly, $R \cup R^{\prime}+u \bar{u}$ is a cycle of length $n+1$ and contains the vertex $v$ and the unique complementary edge. The theorem follows.

Theorem 2.4. Every shortest path between any two distinct vertices in $F Q_{n}$ contains at most one complementary edge.

Proof. Suppose that $P=\left(u, u_{1}, \ldots, u_{i}, \bar{u}_{i}, u_{i+1}, \ldots, u_{j}, \bar{u}_{j}, \ldots, v\right)$ is a shortest $u v$-path in $F Q_{n}$ containing more than one complementary edge, where $\left(u_{i}, \bar{u}_{i}\right)$ and $\left(u_{j}, \bar{u}_{j}\right)$ are the two complementary edges that first occur in $P$ in the order from $u$ to $v$. Then the section $\left(\bar{u}_{i}, \ldots, u_{j}\right)$ of $P$ contains no complementary edge and is a shortest $\bar{u}_{i} u_{j}$-path. Since $d_{H}\left(u_{i}, \bar{u}_{j}\right)=d_{H}\left(\bar{u}_{i}, u_{j}\right)$, the section $\left(u_{i}, \bar{u}_{i}, u_{i+1}, \ldots, u_{j}, \bar{u}_{j}\right)$ of $P$ is not a shortest $u_{i} \bar{u}_{j}$-path, whereby $P$ is not the shortest path, a contradiction. The theorem follows.

Theorem 2.5. Let $u$ and $v$ be two vertices in $F Q_{n}(n \geq 2)$. If $d_{H}(u, v) \leq\left\lfloor\frac{n}{2}\right\rfloor$, then any shortest uv-path in $F Q_{n}$ contains no complementary edges. If $d_{H}(u, v)>\left\lceil\frac{n}{2}\right\rceil$, then any shortest $u v$-path in $F Q_{n}$ contains exactly one complementary edge.

Proof. Suppose $d_{H}(u, v)=h \leq\left\lfloor\frac{n}{2}\right\rfloor$; then $v \neq \bar{u}$ since $d_{H}(u, \bar{u})=n$. Suppose to the contrary that there is a shortest $u v$-path $P$ in $F Q_{n}$ containing a complementary edge. By Theorem $2.4, P$ contains exactly one complementary edge, say $e=(x, \bar{x})$. Let $d_{H}(u, x)=i$ and $d_{H}(\bar{x}, v)=m$. Then $d_{F Q_{n}}(u, x)=d_{H}(u, x)=i<\left\lfloor\frac{n}{2}\right\rfloor, d_{F Q_{n}}(\bar{x}, v)=d_{H}(\bar{x}, v)=m<\left\lfloor\frac{n}{2}\right\rfloor$ and $i+m+1=h \leq\left\lfloor\frac{n}{2}\right\rfloor$. Since $d_{H}(u, x)+d_{H}(u, \bar{x})=n$, we have $d_{H}(u, \bar{x})=n-i$. Also since $d_{H}(u, v)+d_{H}(\bar{x}, v) \geq d_{H}(u, \bar{x})$, 
we can deduce a contradiction as follows:

$$
\left\lfloor\frac{n}{2}\right\rfloor \geq d_{H}(u, v) \geq d_{H}(u, \bar{x})-d_{H}(\bar{x}, v)=n-i-m>n-h \geq\left\lceil\frac{n}{2}\right\rceil .
$$

Thus, $P$ contains no complementary edges.

If $d_{H}(u, v)>\left\lceil\frac{n}{2}\right\rceil$, since $d_{F Q_{n}}(u, v) \leq\left\lceil\frac{n}{2}\right\rceil$, any shortest path between $u$ and $v$ must contain a complementary edge. By Theorem 2.4, the theorem follows.

Remarks. If $n$ is odd and $d_{H}(u, v)=\left\lceil\frac{n}{2}\right\rceil$, then there are a shortest $u v$-path containing no complementary edge and a shortest $u v$-path containing exactly one complementary edge in $F Q_{n}$.

Theorem 2.6. If $F Q_{n}$ contains an odd cycle, then any shortest odd cycle contains exactly one complementary edge and the length is $n+1$.

Proof. If $F Q_{n}$ contains an odd cycle, then by Theorem $2.1 n$ is even and any odd cycle must contain a complementary edge. Moreover, any odd cycle must contain at least one edge that is not a complementary edge since any two complementary edges are not adjacent. Assume that $C=\left(v_{0}, v_{1}, \ldots, v_{2 l}, v_{0}\right)$ is a shortest odd cycle of length $2 l+1$ in $F Q_{n}$, where the edge $e_{l}=\left(v_{l}, v_{l+1}\right)$ is not a complementary edge. Consider two paths in $C, P_{1}=\left(v_{0}, v_{1}, \ldots, v_{l}\right)$ and $P_{2}=\left(v_{l+1}, \ldots, v_{2 l}, v_{0}\right)$. If $P_{1}$ is not shortest in $F Q_{n}$ and $P^{\prime}$ is, then the length of any cycle $C^{\prime}$ in $P_{1} \cup P^{\prime}$ is less than $2 l+1$, so $C^{\prime}$ is an even cycle. Thus, the lengths of $P_{1}$ and $P^{\prime}$ have the same parity. By a similar argument, we can prove that the lengths of $P^{\prime}$ and $P_{2}+e_{l}$ have the same parity. It follows that the lengths of $P_{1}$ and $P_{2}+e_{l}$ have the same parity, which means that $C$ is an even cycle, a contradiction. Therefore, $P_{1}$ is a shortest path. Similarly, $P_{2}$ is also a shortest path. By Theorem 2.4, $C$ contains at most two complementary edges. Since $C$ is an odd cycle, $C$ contains exactly one complementary edge by Theorem 2.2.

By Theorem 2.3, the length of $C$ is at least $n+1$. Also by Theorem 2.3, any complementary edge lies on a cycle of length $n+1$ in $F Q_{n}$. This shows that the minimum length of any odd cycle is exactly $n+1$.

\section{Embedding cycles into $F Q_{n}$}

For convenience, we use $Q_{n-1}^{0}$ and $Q_{n-1}^{1}$ to denote the two $(n-1)$-subcubes of $Q_{n}$ induced by the vertices with the leftmost bit 0 and 1 , respectively. Then, all of $2^{n-1}$ complementary edges of $F Q_{n}$ join $0 u$ and $1 \bar{u}$ between $Q_{n-1}^{0}$ and $Q_{n-1}^{1}$ for any $u \in V\left(Q_{n-1}\right)$.

Lemma 3.1 (Li et al. [9]). If $n \geq 2$, then

(a) for any two different vertices $x$ and $y$ in $Q_{n}$ there exists an $x y$-path of length $l$ with $d_{Q_{n}}(x, y) \leq l \leq$ $2^{n}-1$, where $l$ and $d_{Q_{n}}(x, y)$ have the same parity;

(b) every edge of $Q_{n}$ lies on a cycle of every even length from 4 to $2^{n}$.

Theorem 3.2. Every complementary edge in $F Q_{n}$ lies on a cycle of every even length from 4 to $2^{n}$ for $n \geq 2$.

Proof. Let $e$ be a complementary edge in $F Q_{n}$. Without loss of generality, we may assume that $e=(0 u, 1 \bar{u})$ with $0 u \in V\left(Q_{n-1}^{0}\right)$ and $1 \bar{u} \in V\left(Q_{n-1}^{1}\right)$. Choose an edge $(0 u, 0 v)$ in $Q_{n-1}^{0}$ and a corresponding edge $(1 \bar{u}, 1 \bar{v})$ in $Q_{n-1}^{1}$. We want to prove that the edge $e=(0 u, 1 \bar{u})$ lies on a cycle of length $l$ in $F Q_{n}$ with $4 \leq l \leq 2^{n}$ and $l$ is even. 


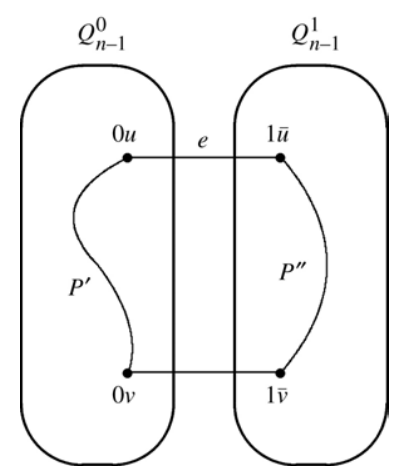

(a) Illustration for

Theorem 3.2.

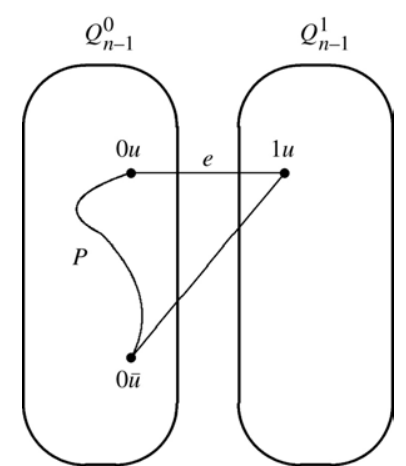

(b) Illustration for case 2 of Theorem 3.3. $n+1 \leq l \leq 2^{n-1}+1$

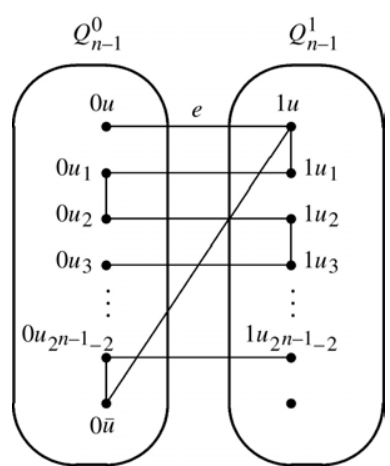

(c) Illustration for case 2 of Theorem 3.3. $2^{n-1}+3 \leq l \leq 2^{n}-1$

Fig. 2. Illustrations for the proofs in Section 3.

We can express $l=l^{\prime}+l^{\prime \prime}+2$ where $1 \leq l^{\prime} \leq 2^{n-1}-1,1 \leq l^{\prime \prime} \leq 2^{n-1}-1$ and both $l^{\prime}$ and $l^{\prime \prime}$ are odd integers. By Lemma 3.1(a), there exist a path $P^{\prime}$ of length $l^{\prime}$ in $\overline{Q_{n-1}^{0}}$ joining $0 u$ and $0 v$ and a path $P^{\prime \prime}$ of length $l^{\prime \prime}$ in $Q_{n-1}^{1}$ joining $1 \bar{u}$ and $1 \bar{v}$. Then $P^{\prime}+(0 v, 1 \bar{v})+P^{\prime \prime}+(1 \bar{u}, 0 u)$ is a cycle containing the edge $e$ in $F Q_{n}$, whose length is equal to $l$ (see Fig. 2(a)). The theorem follows.

Theorem 3.3. If $n$ is odd, then every edge of $F Q_{n}$ lies on a cycle of every even length from 4 to $2^{n}$ for $n \geq 3$. If $n$ is even, then every edge of $F Q_{n}$ lies on a cycle of every even length from 4 to $2^{n}$ and every odd length from $n+1$ to $2^{n}-1$.

Proof. If $n$ is odd, then $F Q_{n}$ is a bipartite graph by Theorem 2.1. By Lemma 3.1(b) and Theorem 3.2, the first assertion follows. We now prove the second assertion.

If $n$ is even, then $F Q_{n}$ is not a bipartite graph by Theorem 2.1. By Lemma 3.1(b) and Theorem 3.2, every edge lies on a cycle of every even length from 4 to $2^{n}$ inclusive.

We now show that every edge of $F Q_{n}$ lies on a cycle of any odd length $l$ with $n+1 \leq l \leq 2^{n}-1$ when $n$ is even. Let $e$ be any edge in $F Q_{n}$. There are two cases.

Case 1 . The edge $e=(u, \bar{u})$ is a complementary edge. In this case, $u$ and $\bar{u}$ belong to the same part of a bipartition of $Q_{n}, d_{Q_{n}}(u, \bar{u})=n$ and $\frac{1}{2}((l-1)-n)=\frac{1}{2}\left((l-1)-d_{Q_{n}}(u, \bar{u})\right)$ is an integer. By Lemma 3.1(a), there is a path of length $l-1$ joining $u$ and $\bar{u}$ with $n \leq(l-1) \leq 2^{n}-2$. Choose $P$ to be such a path of even length $(l-1)$ with $n \leq(l-1) \leq 2^{n}-2$ in $Q_{n}$. Thus, $P+e$ is an odd cycle of length $l$ in $F Q_{n}$ with $n+1 \leq l \leq 2^{n}-1$.

Case 2. The edge $e=(u, v)$ is not a complementary edge. In this case, $u$ and $v$ belong to different parts of a bipartition of $Q_{n}$. Without loss of generality, we may assume $e=(0 u, 1 u)$; then $(1 u, 0 \bar{u})$ is a complementary edge in $F Q_{n}$ and $d_{H}(0 u, 0 \bar{u})=n-1$.

Since $l$ and $(n-1)$ are odd, $\frac{1}{2}((l-2)-(n-1))=\frac{1}{2}\left((l-2)-d_{Q_{n}}(0 u, 0 \bar{u})\right)$ is an integer. By Lemma 3.1(a), there is a path $P$ of odd length $(l-2)$ joining $0 u$ and $0 \bar{u}$ in $Q_{n-1}^{0}$ with $n-1 \leq(l-2) \leq$ $2^{n-1}-1$. In particular, we use $P_{0}$ to denote such a path of length $2^{n-1}-1$ joining $0 u$ and $0 \bar{u}$ in $Q_{n-1}^{0}$.

If $n+1 \leq l \leq 2^{n-1}+1$, then $P+(0 u, 1 u, 0 \bar{u})$ is an odd cycle of length $l$ containing the edge $e$ in $F Q_{n}$ with $n+1 \leq l \leq 2^{n-1}+1$ (see Fig. 2(b)).

If $2^{n-1}+3 \leq l \leq 2^{n}-1$, let $l^{\prime}=l-2^{n-1}-1$; then $2 \leq l^{\prime} \leq 2^{n-1}-2$. We may assume $P_{0}=\left(0 u, 0 u_{1}, 0 u_{2}, \ldots, 0 u_{2^{n-1}-2}, 0 \bar{u}\right)$. For every edge $\left(0 u_{i}, 0 u_{i+1}\right)$ in $\bar{Q}_{n-1}^{0}$, there is a corresponding 
edge $\left(1 u_{i}, 1 u_{i+1}\right)$ in $Q_{n-1}^{1}$. Thus $C=\left(0 u, 0 u_{1}, 1 u_{1}, 1 u_{2}, 0 u_{2}, 0 u_{3}, 1 u_{3}, \ldots, 0 u_{l^{\prime}-1}, 1 u_{l^{\prime}-1}, 1 u_{l^{\prime}}, 0 u_{l^{\prime}}\right.$, $\left.0 u_{l^{\prime}+1}, 0 u_{l^{\prime}+2}, \ldots, 0 u_{2^{n-1}-2}, 0 \bar{u}, 1 u, 0 u\right)$ is a cycle containing the edge $e$ in $F Q_{n}$ whose length is equal to $2^{n-1}+1+l^{\prime}=l$ (see Fig. $\left.2(\mathrm{c})\right)$.

The theorem follows.

\section{Conclusion}

In this work, we obtain some new properties of $F Q_{n}$. We show that every edge of $F Q_{n}$ lies on a cycle of every even length from 4 to $2^{n}$ for $n \geq 2$; furthermore, every edge of $F Q_{n}$ also lies on a cycle of every odd length from $n+1$ to $2^{n}-1$ if $n$ is even. The result that an odd cycle can be embedded into $F Q_{n}$ when $n$ is even shows that $F Q_{n}$ is superior to $Q_{n}$ in view of the cycle embedding capability.

\section{Acknowledgements}

The authors would like to thank the anonymous referees for their helpful comments and suggestions which considerably improved the present version of the work.

\section{References}

[1] Y. Saad, M.H. Schultz, Topological properties of hypercubes, IEEE Trans. Comput. 37 (7) (1988) 867-872.

[2] J. Xu, Topological Structure and Analysis of Interconnection Networks, Kluwer Academic Publishers, Dordrecht, Boston, London, 2001.

[3] A. El-Amawy, S. Latifi, Properties and performance of folded hypercubes, IEEE Trans. Parallel Distrib. Syst. 2 (1991) 31-42.

[4] S.C. Liaw, G.J. Chang, Generalized diameters and Rabin numbers of networks, J. Comb. Optim. 2 (1998) $371-384$.

[5] E. Simó, J.L.A. Yebra, The vulnerability of the diameter of folded $n$-cubes, Discrete Math. 174 (1997) 317-322.

[6] D.R. Duh, G.H. Chen, J.F. Fang, Algorithms and properties of a new two-level network with folded hypercubes as basic modules, IEEE Trans. Parallel Distrib. Syst. 6 (7) (1995) 714-723.

[7] C.N. Lai, G.H. Chen, D.R. Duh, Constructing one-to-many disjoint paths in folded hypercubes, IEEE Trans. Comput. 51 (1) (2002) 33-45.

[8] D. Wang, Embedding Hamiltonian cycles into folded hypercubes with faulty links, J. Parallel Distrib. Comput. 61 (2001) 545-564.

[9] L.K. Li, C.H. Tsai, J.M. Tan, L.H. Hsu, Bipanconnectivity and edge-fault-tolerant biancyclicity of hypercubes, Inform. Process. Lett. 87 (2003) 107-110. 